\title{
NMR analysis on microfluidic devices by remote detection
}

\author{
Erin E. McDonnell, SongI Han*, Christian Hilty, Kimberly Pierce, and Alexander Pines
}

Lawrence Berkeley National Laboratory, Materials Sciences Division, and University of California Berkeley, Department of Chemistry, Berkeley CA 94720, USA

*Present address: Department of Chemistry and Biochemistry, University of California Santa Barbara, CA 93106-9510, USA

\begin{abstract}
We present a novel approach to perform high-sensitivity NMR imaging and spectroscopic analysis on microfluidic devices. The application of NMR, the most information rich spectroscopic technique, to microfluidic devices remains a challenge because the inherently low sensitivity of NMR is aggravated by small fluid volumes leading to low NMR signal, and geometric constraints resulting in poor efficiency for inductive detection. We address the latter by physically separating signal detection from encoding of information with remote detection. Thereby, we use a commercial imaging probe with sufficiently large diameter to encompass the entire device, enabling encoding of NMR information at any location on the chip. Because large-diameter coils
\end{abstract}


are too insensitive for detection, we store the encoded information as longitudinal magnetization and flow it into the outlet capillary. There, we detect the signal with optimal sensitivity using a solenoidal microcoil, and reconstruct the information encoded in the fluid. We present a generally applicable design for a detection-only microcoil probethat can be inserted into the bore of a commercial imaging probe. Using hyperpolarized ${ }^{129} \mathrm{Xe}$ gas, we show that this probe enables sensitive reconstruction of NMR spectroscopic information encoded by the large imaging probe while keeping the flexibility of a large coil.

Keywords: microfluidics, microcoil, NMR, remote detection, flow

\section{Introduction}

Microfluidic devices are being developed and used with increasing success in chemical and biological sciences, performing functions that previously required elaborate instrumentation. ${ }^{1,2}$ Applications are as varied as the analysis of biological macromolecules ${ }^{3}$, chemical reactors ${ }^{4}$, and sensing in the gas phase. ${ }^{5}$ Miniaturized devices hold several advantages over standard laboratory methods. For example, they require little reactant and produce little waste, addressing both economic and environmental concerns. Processes can be automated and repeated with a high degree of precision, and many steps can be combined on one chip. Such microTotal Analysis Systems $(\mu \mathrm{TAS})^{6}$ eliminate manual handling and thus reduce errors, while still increasing speed and efficiency ${ }^{6-8}$. On the other hand, when working with microfluidic systems, new challenges are faced, for example coupling to the macroscopic world. The techniques for signal detection, in particular, have to be adapted to conform to the miniaturized size and geometries of the channels. 
So far, detection methods that have been successfully applied with microfluidic devices for analysis include chemiluminescence, electrochemiluminescence, electrochemical detection, UV-visible absorption detection, fluorescence, mass spectrometry, and $\mathrm{NMR}^{9-13}$. Of these, only NMR and mass spectrometry will reveal direct structural information about the products. NMR has the added advantage that it is noninvasive, while being capable of providing a wider range of physiochemical information about the flowing analyte, e.g. the conformation of molecules and the composition of the reaction mixture as well as diffusion characteristics and flow maps. Due to these benefits, many different applications for NMR with microfluidics have been proposed. For example, NMR has been coupled with $\mu$ TAS HPLC and capillary electrophoresis ${ }^{14-16}$, used to image cells in a capillary ${ }^{17}$, and to study protein folding ${ }^{18}$ as well as chemical reactions on a microscale ${ }^{19}$. These studies were entirely conducted in capillaries, around which a highly sensitive solenoidal microcoil was wrapped. For kinetic processes, either a variation of the flow rate ${ }^{20}$ or the placement of multiple coils at different positions ${ }^{14,21,22}$ was used to obtain spectra from different reaction time points. Due to a wealth of functions that can only be performed by more complex microfluidic chip devices, it is of great interest to make the powerful NMR analysis technique applicable to chip devices as well. Recently, microfabricated surface microcoils have been integrated directly onto microfluidic chip devices in specific locations where a spectrum is desired ${ }^{23-25}$. This enables spectroscopy to be performed on a predefined location on a microfluidic chip, yet due to their geometries, these coils have a low filling factor and in terms of sensitivity cannot compete with a solenoidal microcoil. While successfully applicable in many cases, NMR detection with solenoidal coils used with capillaries, as well as microfabricated surface coils with chip devices, lacks flexibility in its application. 
We seek an approach which allows NMR spectroscopy and imaging from a spatial location of choice on a microfluidic chip device without a new design or modification in the setup and without employing multiple microcoils, each of which has to be placed on or inside the chip. Naturally, a large coil encompassing the entire device would fulfill these criteria. However, the filling factor of such a coil will always be prohibitively small, as the fluid sample in a microfluidic device fills a very small portion of the total volume, thus preventing the use of such a coil for NMR detection. This limitation can be overcome by using remote detection, a recently introduced technique that allows separate optimization of the encoding and the detection hardware for NMR spectroscopy and imaging experiments ${ }^{26,27}$. Remote detection allows us to use a large rf coil and 3D gradient coils so that homogeneous rf and gradient fields are applied to the entire volume for encoding NMR spectral or image information. Then, instead of directly detecting the signal at this location of information encoding, the principle of indirect detection is employed using physically-separated detection hardware. The NMR time domain signal originating from the precessing transverse magnetization is stored after incremented evolution times as long-living longitudinal magnetization in the spin system. Subsequently, the encoded spins that carry a characteristic magnetization amplitude, flow with the solution out of the microfluidic device into the outlet capillary. There, the magnetization is detected with optimal sensitivity using a micro-solenoid coil as the detection hardware. This procedure is repeated multiple times for each evolution time increment and the corresponding amplitudes are detected. The collected amplitudes compose the NMR signal that contains the desired NMR information from the encoding location of choice. Complex NMR signal can be obtained by repeating each reconstruction twice in a phase-sensitive fashion ${ }^{26}$. The concept of remote detection is ideal for application to microfluidic chip devices because it allows us to 
employ both the versatility and space of a large imaging coil setup and the optimized filling factor, and therefore sensitivity, of a small micro-coil probe ${ }^{28,29}$.

\section{Experimental Section: two-probe design for remote detection}

A detection-only micro-solenoid coil probe was designed for remote detection of NMR information about a microfluidic device that is encoded using a commercially available imaging probe (Varian, Palo Alto, CA, USA). The clear bore of the imaging probe allows insertion of the specifically designed detection probe (diameter $36 \mathrm{~mm}$ at base, $29 \mathrm{~mm}$ at head; length $560 \mathrm{~mm}$ ) such that the microcoil is positioned less than a centimeter below the lower edge of the imaging coil. This design principle is illustrated in Figure 1a. Many commercial imaging probes have clear bores of 15-30 mm diameter, making them suitable for remote detection using the presented type of detector.

The central piece of the detection probe is a micro-solenoid coil, which was turned by hand from $130 \mu \mathrm{m}$ diameter copper wire with insulation coating onto a polyimide sleeve $(487 \mu \mathrm{m}$ i.d./537 $\mu \mathrm{m}$ o.d.; Upchurch Scientific, Inc. Oak Harbor, WA). The coil was then placed in an enclosing Teflon support holding it perpendicular to the main magnetic field (Figure 1b). It is $2.9 \mathrm{~mm}$ long and has an inner diameter of $400 \mu \mathrm{m}$, fitting a Teflon capillary of $150 \mu \mathrm{m}$ i.d./350 $\mu \mathrm{m}$ o.d (Upchurch Scientific, Inc. Oak Harbor, WA) or a polyimide capillary of $310 \mu \mathrm{m}$ i.d./360 $\mu \mathrm{m}$ o.d. (Cole-Parmer Instrument Company, Vernon Hills, IL), either of which can be used for fluid transfer to and from the microfluidic device. It was crucial to position the detection and the encoding coils as close together as possible due to the limited extent of the homogeneous field region of the superconducting NMR magnet. This was achieved by the narrow cylindrical support for the coil shown in Figure 1b, which was machined out of Delrin and mounted on top of the probe body. Due 
to the proximity of the two coils, a removable copper shield was placed over the entire microcoil and support assembly and was well grounded to the rest of the probe. If the detection probe were to be adapted for use with different imaging probes, the cylindrical support for the coil and its shield may be the only part that has to be customized. Crosstalk between the detection and encoding coils was thus reduced to a level not detectable by a conventional frequency analyzer, allowing the acquisition of artifact-free remotely detected NMR spectra. The fluid carrying capillary was led from the encoding to the detection coil through a small hole in the copper shielding.

For initial experiments, to demonstrate the applicability of remotely detected NMR to microfluidic gas flow, a xenon gas mixture was used $\left(0.3 \%\right.$ of NMR-active ${ }^{129} \mathrm{Xe}$ in a mixture of $1 \% \mathrm{Xe}$ at natural isotope abundance, $89 \% \mathrm{He}$ and $10 \% \mathrm{~N}_{2}{ }^{30}$ ). Xenon was hyperpolarized to a level of approximately 5\% in a polarizer (former MITI, Amersham, Durham, NC) using spin-exchange optical pumping of rubidium vapor. The detection micro probe was tuned to the frequency of ${ }^{129} \mathrm{Xe}$ (82.9 MHz) in a 7 T NMR magnet. Variable capacitors (Polyflon, Norwalk, CT) were placed on a support in the probe body located below the coil, forming a standard circuit ${ }^{31}$ for tuning (Polyflon $5-45 \mathrm{pF}$ capacitor) and for impedance matching to $50 \Omega$ (Polyflon 1-10 pF in series with American Technical Ceramics $10 \mathrm{pF}$ capacitor). The quality factor ${ }^{31}$ of this probe was measured to be 52 , and it has a $90^{\circ}$ pulse length of $0.8 \mu \mathrm{s}$ at the rf power used for the experiments. In contrast, the imaging probe has a $90^{\circ}$ pulse length of $120 \mu$ s at the same rf power. Based on these values, the remote detection of signal affords a sensitivity gain of a factor of 150 for systems where $T_{1}$ relaxation during transfer is negligible. This translates into a gain in measurement time required to acquire a spectrum of more than a factor of 20,000, again illustrating that the large coil is by far not suitable for signal detection. 


\section{Results and Discussion}

Initial experiments to measure the flow in microfluidic devices, as well as proof-of-pinciple spectra were acquired using hyperpolarized xenon gas. An experimental scheme to obtain timescales for travel times of fluid moving between the two coils within microfluidic channels is shown in Figure $2^{26}$. After the spin volume of choice has been tagged with the imaging coil, the batches of signal are detected with the microcoil as they arrive. The tagging is implemented by applying an inversion rf pulse to perturb the longitudinal magnetization of the fluid batch in the first coil and the detection by a train of read-out $\left(90^{\circ}\right)$ rf pulses, which yields resolution as a function of the time of travel.

Travel-time curves obtained by the sequence in Figure 2 are displayed in Figure 3, for two different flow rates. The sample consisted of a polyimide capillary that transversed the imaging coil and then ran through the detection coil. At short travel-times, the tagged fluids have not reached the detector, which is why signal is detected from unperturbed fluid batches at that point, i.e. maximum signal intensity is measured at the detector. The dips at longer travel times represent the detection of fluid samples with perturbed magnetization, i.e. fluid sample that was present in the encoding coil at the time of the inversion. These travel times not only show the time scale of flow but also the spreading of the sample during the travel distance, which is a direct consequence of the flow properties resulting from the capillary arrangements or channel geometry of the chip. When comparing the curves of Figure $3 \mathrm{a}$ and $\mathrm{b}$, it is apparent that at the slower rate, the encoded signal arrives at the detection coil later and also remains longer in the detection coil. Furthermore, the asymmetry in the dips is a direct consequence of a non-uniform flow profile across the channel diameter. 
Once the time of travel is known, and the flow distribution understood, the sequence in Figure $4^{26}$ can be used to acquire a remotely detected spectrum of the fluid in the chip. The spin volume of choice is excited in the encoding coil and allowed to evolve according to their chemical shift for the incremented time $t_{1}$ before being stored as transverse magnetization along the longitudinal axis. The stored magnetization will then be available to "carry" the encoded information for the amount of time dictated by $T_{1}$, the lifetime of longitudinal magnetization. After waiting for the calibrated travel time $\left(\tau_{\text {travel }}\right)$, the spins are detected in one or several batches either incorporating all spins that were in the encoding coil during encoding, or a subset thereof. Each value for the magnetization is a point in the remotely reconstructed free induction decay (see inset in Figure 5a), where the time axis corresponds to the amount of time the encoded spins were allowed to evolve. Fourier transformation of this data yields the remotely detected spectrum within the encoding coil (Figure 5a). In this way, a spectrum representing the chemistry of the sample in the microfluidic device can be obtained while still taking advantage of the sensitive detection coil.

Spatial selectivity within the chip can be achieved most easily by detecting only a subset of the excited spins. The proper timing of detection will provide spatial selection of the volume of interest. Optionally, for increased precision of localizing a specific point, the hard pulses in the sequence of Figure 4 can be replaced by shaped pulses concomitant with the application of gradients for slice selection ${ }^{32}$.

The advantage of using remote detection with the current setup is visualized by the trace in Figure $5 \mathrm{~b}$, which is a spectrum acquired directly with the encoding coil in the conventional manner. As expected, due to a filling factor of approximately $10^{-5}$ in this coil, no signal could be detected even when using 17,000 scans. On the other hand, the quality of the remotely detected spectrum in Figure $5 \mathrm{a}$ is comparable to that of a directly detected spectrum of xenon gas in the capillary, using 
the detection coil. The remotely detected spectrum, which was reconstructed from one acquisition in the detection coil for each increment of $t_{1}$, corresponds to the spectrum of a sample region in the encoding coil with equal volume to a sample in the detection coil, which, for the polyimide tubing, is $219 \mathrm{nl}$. This spectrum was acquired with $100 \mathrm{t}_{1}$ increments, and 16 transients per increment. It has a line width of $125 \mathrm{~Hz}$, which is at the digital resolution for the acquisition parameters that were used, and a signal-to-noise ratio of 7.7. This translates to a theoretical signal-to-noise ratio per scan of 0.19 .

The comparison to a spectrum of the same amount of sample that was directly acquired in the detection coil (Figure 5c) is well within the limits of practical usefulness. That spectrum was acquired to the same digital resolution, and is also showing a line width of $125 \mathrm{~Hz}$. The width of this line can primarily be explained by the reduced residence time in the coil due to the flow. Its signal-to-noise ratio for 500 acquired transients is 12.7 , corresponding to a theoretical signal-tonoise ratio per scan of 0.56 . This means that in this particular case, based on the number of transients and the sample volume the remote detection of signal was a factor of 3 less efficient than the theoretical maximum sensitivity, which is given by the signal-to-noise ratio of a directly acquired spectrum using the detection coil. Yet on the other hand, the achieved sensitivity gain still translates to a factor of approximately 150 in signal-to-noise ratio when comparing to the direct acquisition of a spectrum in the encoding coil as discussed before by means of the $90^{\circ}$ pulse length comparison. The loss in signal compared to the theoretical maximum that would be achievable with direct detection with a solenoidal coil can be explained by several factors. One of them is the spreading of the gas as it flows through the capillary between the two coils due to the characteristics of laminar flow, which is experimentally evidenced by the slightly non-symmetrical appearance of the spin travel profiles of Figure 3. Another factor is the decrease of the detected 
signal amplitude due to $T_{1}$ relaxation during the travel period. Also, in the remote detection scheme, some amount of signal is inevitably lost due to the presence of $T_{2}$ relaxation during both the encoding and the detection step, while a directly acquired spectrum is subject to the effect of $\mathrm{T}_{2}$ relaxation only during detection.

The experimental time for the remotely detected spectrum was longer than for the directly detected spectrum because with the pulse sequence that was used (Figure 3), the total length in time of each transient, or repetition rate, is determined by the clearance time through the detection coil of all of the fluid in the encoding coil. This is by no means necessary when acquiring a spectrum from a specific location on the chip. Once the time of travel is known, it is in principle possible to modify the pulse sequence in Figure 4 to use interleaved excitation and acquisition. Thereby, a next batch of spins is already encoded before a previous batch has actually reached the detection coil. This would enable the use of repetition rates that are only slightly lower than for a directly acquired spectrum, thus making the experimental time between a remotely and a directly detected spectrum almost equal.

The ability to use remote detection with the large encoding coil offers the advantage of complete flexibility for the location and sample size of on-chip spectroscopy, while nearly retaining the sensitivity of a solenoidal microcoil. Furthermore, this method retains the favorable properties of optimal $\mathrm{B}_{0}$ and $\mathrm{B}_{1}$ homogeneity of the larger coil. When compared with the use of micro-surface coils that are conventionally used for on-chip spectroscopy, which suffer both from lower sensitivity than a solenoidal coil and from reduced field homogeneity when compared to a large coil, it appears plausible that the proposed method of remote spectroscopy on microfluidic devices is favorable in many cases. Therefore, the presented approach may become the analysis method of choice for many microfluidic applications. 
For the current detection probe, a micro solenoid coil was chosen due to its optimal sensitivity and ease of fabrication. This is not a condition; it would also be possible to achieve sensitive detection with vertical bore rf coils, which could make the implementation of the capillary outlet of chip devices more convenient. Possibilities are small saddle coils, which however are more difficult to manufacture, or toroid cavities ${ }^{33}$, which do not show reduced mass sensitivity propotional to the diameter.

When using hyperpolarized xenon for NMR with microfluidics, the absolute limit of the number of spins that can be detected by NMR is dramatically reduced. For example, the spectrum in Figure 5c was taken of $33.8 \mathrm{pmol}$ spins at a time in a volume of $51.2 \mathrm{nl}$. Based on this, the theoretical limit of detection per scan, which we define for this purpose as the number of spins yielding a signal-to-noise ratio of 2 , is extrapolated to be 320 pmol.

However, it is important to note that the presented method is not limited to hyperpolarized gases, but can be applied just as easily with other fluids such as liquids and dissolved solutes. A condition is that the time of travel between the detection and the encoding location is shorter than approximately one or two times the spin-lattice relaxation time $\left(T_{1}\right)$. This will be the case for example for many applications in chemistry, which involve small molecules that generally have relaxation times on the order of seconds to tens of seconds. The travel time for flow out of miniaturized devices into adjacent outlet capillaries can be easily in the subsecond range, as can be seen in the examples presented in Figure 2. Depending on the nature of the system studied, the signal-to-noise ratio will in these cases be larger or smaller than what was obtained here with hyperpolarized xenon. For example, the protons of water would yield a sensitivity that is approximately 30 times larger than that of xenon gas under the conditions used. From this number, 
the sensitivity for a solute at any concentration can be extrapolated; a signal level equal to the spectra presented here would be achieved with a substance of approximately $30 \mathrm{mM}$ concentration.

\section{Conclusion}

We have designed experiments which allow full versatility in NMR spectroscopy and imaging analysis on a microfluidic chip. Using a novel two-probe setup, we have remotely acquired flow and spectroscopic information about a sample of hyperpolarized xenon in a $310 \mu \mathrm{m}$ i.d. $/ 360 \mu \mathrm{m}$ o.d. capillary encased in a 30mm i.d. imaging coil by means of a second detector-only micro-solenoid coil. As expected, the attempt for direct signal detection of the sample using the imaging coil was unsuccessful. However, the designed perturbation using the imaging coil-either inversion of magnetization or encoding of spectroscopic information - could be successfully observed by remote detection. Furthermore, other means of spin manipulation such as slice selection and spatial encoding are straightforward and have been demonstrated previously. ${ }^{27}$ These results demonstrate the feasibility of this technique for determining information about the sample from any point in microfluidic chip devices and obtaining flow information through the chip device. The two-probe remote detection approach can be generally adapted to monitor reactions, evaluate chip elements such as pumps and valves, and analyze the structures of products. Furthermore, the implementation of this novel approach is straightforward, only requiring a simple detector-only probe to be manufactured and adapted to a commercial imaging probe.

\section{ACKNOWLEDGEMENTS}

The authors thank Rachel Martin for valuable discussions and hardware advice. This work was supported by the Director, Office of Science, Office of Basic Energy Sciences, Materials Sciences 
Division of the US Department of Energy under Contract No. DE-AC03-76SF00098. C.H. acknowledges support from the Schweizerischer Nationalfonds through a post-doctoral fellowship. 
FIGURE CAPTIONS
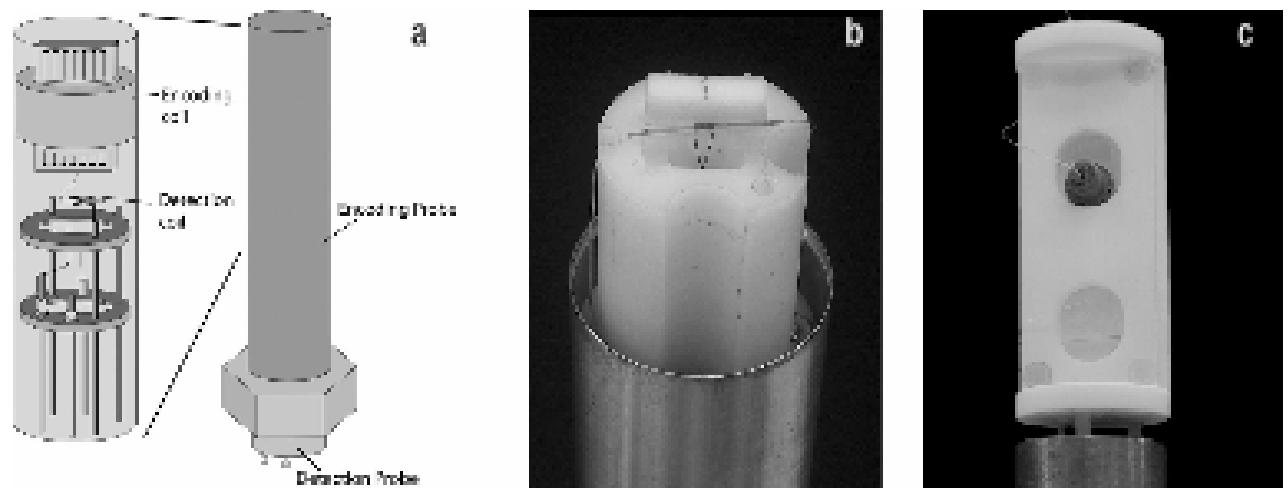

Figure 1: a. A diagram of the two-probe setup for NMR remote detection of microfluidics. An imaging probe with a $30 \mathrm{~mm}$ i.d. coil is used to encode information into the analyte flowing inside of a microfluidic chip device. Below the encoding coil, a second probe with a microcoil is placed, making use of the clear bore inside the imaging probe. A capillary with $350 \mu \mathrm{m}$ outer diameter leads the fluid from the outlet of the microfluidic device to the detection coil. b. A photograph of the top of the detection probe, with its copper shield removed. At the top, the image shows the horizontal microcoil (2.9 $\mathrm{mm}$ long, $400 \mu \mathrm{m}$ inner diameter) on its supporting polyimide sleeve, together with the rear piece of the encasing teflon support. The rest of the image is a machined Delrin cylinder mounted on the probe body, which positions the microcoil less than $1 \mathrm{~cm}$ below the encoding coil of the imaging probe. c. A photograph of a microfluidic chip holder which can be placed on top of the detection coil to hold a chip in place in the center of the imaging coil. 


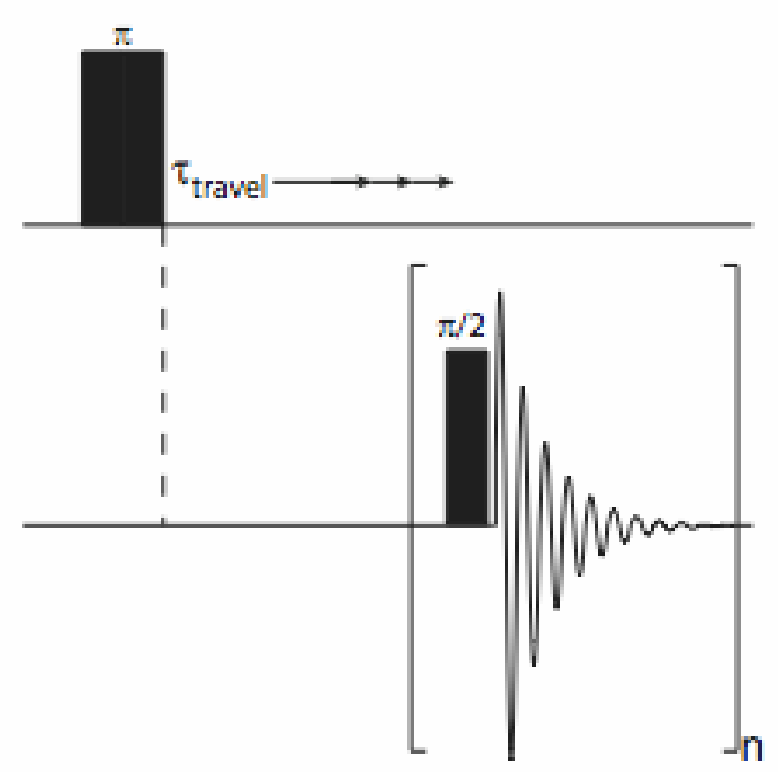

Figure 2: Experiment for measuring the time of travel between the encoding and detection coils. The upper line represents pulse sequence elements applied to the encoding coil, and the lower line elements applied to the detection coil. $90^{\circ}$ pulse lengths are $80 \mu$ s for the encoding coil and $0.8 \mu \mathrm{s}$ for the detection coil. The actual $\mathrm{rf}$ power on the detection coil is $3 \mathrm{~dB}$ lower than on the encoding coil. All pulse phases are $\mathrm{x}$. The acquisition length for one individual FID detected from the encoding coil is set at or below the residence time for fluid in the coil. The number of repetitions of the detection element, $\mathrm{n}$, is set so that the total acquisition length covers all possible times of travel for fluid between the encoding and detection coil. 


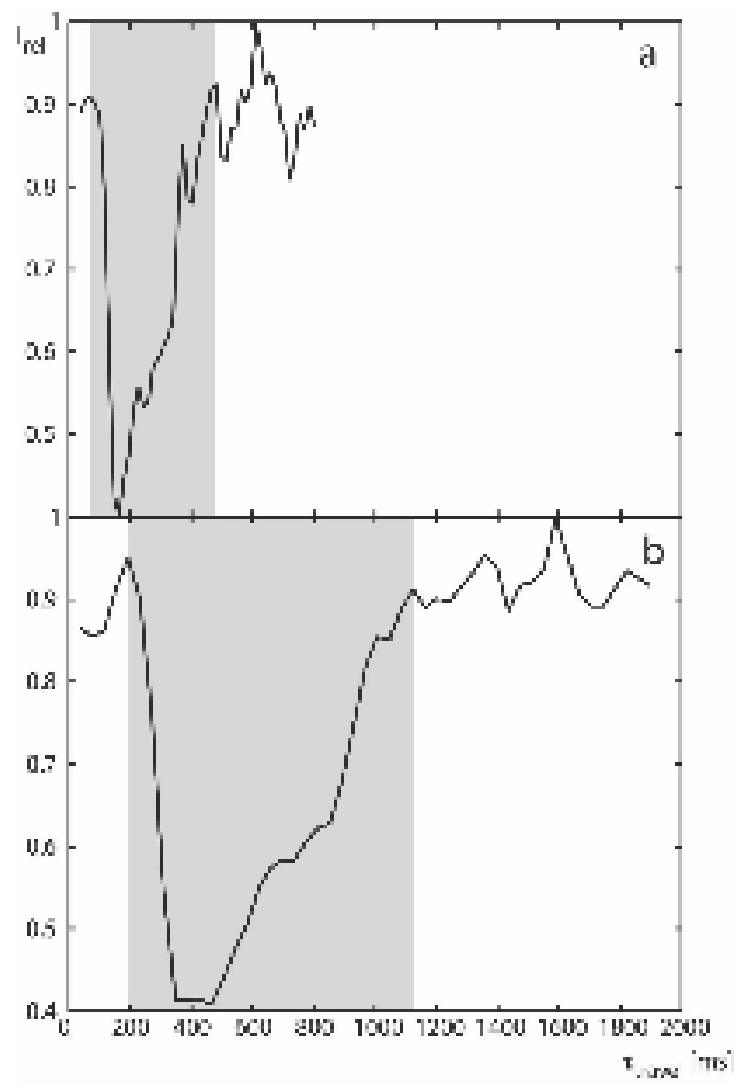

Figure 3: Experiments showing the amount of time it takes for a sample of ${ }^{129} \mathrm{Xe}$ to travel from the encoding to the detection coil at two different flow rates, a, $14.6 \mu 1 / \mathrm{s}$ and $\mathrm{b}, 5.47 \mu \mathrm{l} / \mathrm{s}$ measured at $23^{\circ} \mathrm{C}$ and $1 \mathrm{~atm}$. The pulse sequence of Figure 2 was used to acquire the data. The dip in the curve shows the times over which the encoded sample arrives in the detection coil. Both spectra were acquired with 500 scans and a spectral width in the direct dimension of $100 \mathrm{kHz}$. The data in a took 7 minutes to acquire, and $\mathrm{b}$ took 16 minutes. In a, the sample flow rate was measured at the outlet as twice that of $b$. The residence times for the samples in the encoding coil are $375 \mathrm{msec}$ (top) and $925 \mathrm{msec}$ (bottom). 


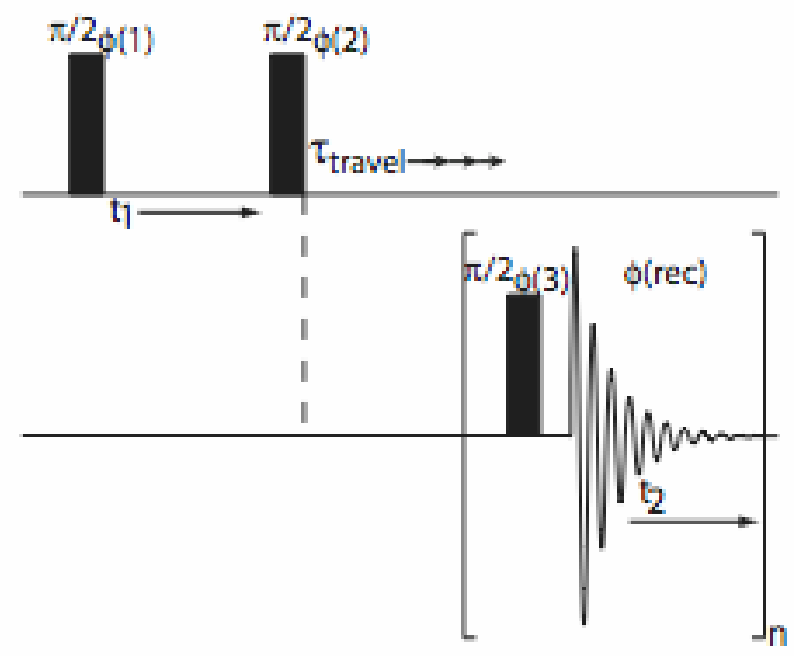

Figure 4: Experiment for remotely reconstructing an NMR spectrum from within a microfluidic chip inside of the encoding coil. As in Figure 2, the upper line represents pulse sequence elements applied to the encoding coil, and the lower line elements applied to the detection coil. Phases are $\phi_{1}$ $=-\phi_{2}=\phi_{3}=\phi_{\mathrm{rec}}=\{\mathrm{x},-\mathrm{x}, \mathrm{y},-\mathrm{y}\}$, with all the other parameters being the same as in Figue 2. For simplicity of setup, it is recommended to acquire all FIDs, but to use only the FIDs corresponding to actual travel times for signal reconstruction. Alternatively, when optimizing the time required for acquiring the remotely detected spectrum, the encoding and acquisition may be done in an interleaved manner (see text). 


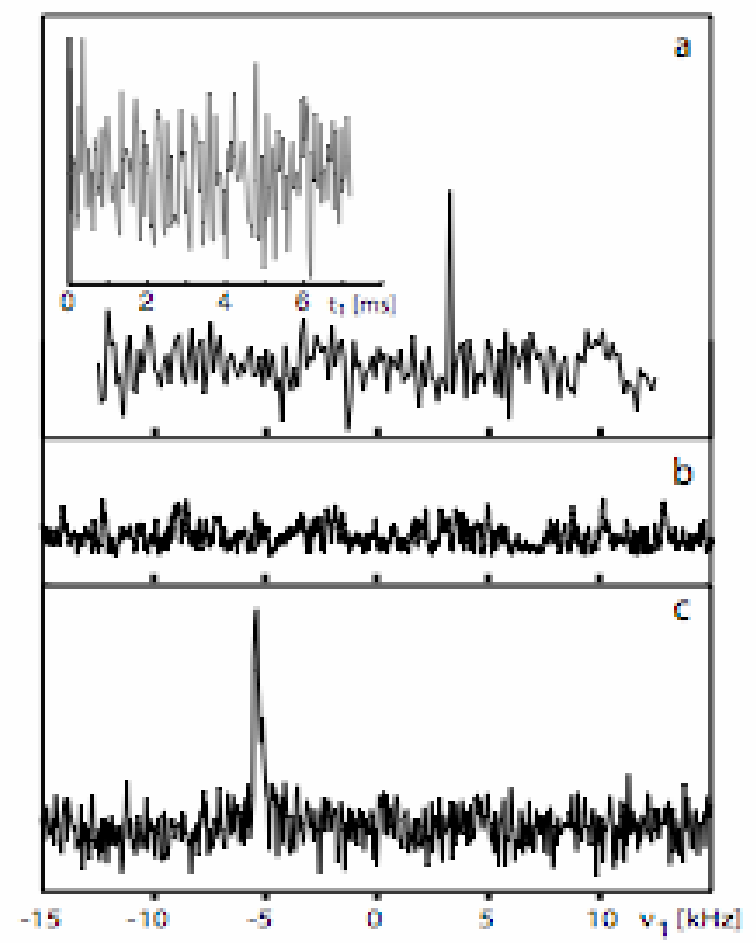

Figure 5: Three spectra collected from a $150 \mu \mathrm{m}$ i.d. capillary placed in the center of the encoding coil and through the detection coil (see Figure 1) a. A remote spectrum encoded in the imaging coil and detected with a $400-\mu \mathrm{m}$ diameter solenoid in the detection probe. The remotely reconstructed free induction decay is inset. The remote fid was reconstructed from 100 points and averaged over 16 transients, with spectral widths in the direct and indirect dimensions of $100 \mathrm{kHz}$ and $25 \mathrm{kHz}$ respectively. The sample was hyperpolarized xenon in polyimide tubing. The entire acquisition took 20 minutes. b. A spectrum acquired directly from the encoding coil (30 $\mathrm{mm}$ i.d., $40 \mathrm{~mm}$ in height). Signal was averaged over 17,000 scans with a spectral width of $100 \mathrm{kHz}$. The spectrum took 45 minutes to acquire. The sample was hyperpolarized xenon $\left(0.3 \%\right.$ of NMR-active ${ }^{129} \mathrm{Xe}$ in a mixture of $1 \% \mathrm{Xe}$ at natural isotope abundance, $89 \% \mathrm{He}$ and $10 \% \mathrm{~N}_{2}$ ) in Teflon tubing. c. A directly acquired spectrum obtained using the solenoidal detection coil over 500 transients and a spectral width of $100 \mathrm{kHz}$. It took 45 seconds. The sample was hyperpolarized xenon in polyimide 
tubing. In all cases, the flow rate of hyperpolarized xenon was $\sim 0.3 \mathrm{~mL} / \mathrm{min}$ measured at $23^{\circ} \mathrm{C}$ and $1 \mathrm{~atm}$. 


\section{REFERENCES}

(1) Figeys, D.; Pinto, D. Anal. Chem. 2000, 72, 330.

(2) Chovan, T.; Guttman, A. Trends Biotechnol. 2002, 20, 116-122.

(3) Burns, M. A.; Johnson, B. N.; Brahmasandra, S. N.; Handique, K.; Webster, J. R.;

Krishnan, M.; Sammarco, T. S.; Man, P. M.; Jones, D.; Heldsinger, D.; Mastrangelo, C. H.;

Burke, D. T. Science 1998, 282, 484-487.

(4) Kobayashi, J.; Mori, Y.; Okamoto, K.; Akiyama, R.; Ueno, M.; Kitamori, T.; Kobayashi, S. Science 2004, 304, 1305-1308.

(5) Ueno, Y.; Horiuchi, T.; Morimoto, T.; Niwa, O. Anal. Chem. 2001, 73, 4688-4693.

(6) Reyes, D. R.; Iossifidis, D.; Auroux, P.-A.; Manz, A. Anal. Chem. 2002, 74, 2623-2636.

(7) Mitchell, P. Nat. Biotechnol. 2001, 19, 717-721.

(8) Liu, J.; Hansen, C.; Quake, S. R. Anal. Chem. 2003, 75, 4718-4723.

(9) Auroux, P.-A.; Iossifidis, D.; Reyes, D. R.; Manz, A. Anal. Chem. 2002, 74, 2637-2652.

(10) Lacey, M. E.; Tan, Z. J.; Webb, A. G.; Sweedler, J. V. J. Chromatogr. A 2001, 922, 139149.

(11) Schwarz, M. A.; Hauser, P. C. Lab Chip 2001, 1, 1-6.

(12) Li, P. d. C., L; Cai, J; Sangar, M Lab Chip 2004, 4, 174-180.

(13) Kameoka, J.; Craighead, H. G.; Zhang, H.; Henion, J. Anal. Chem. 2001, 73, 1935-1941.

(14) Wolters, A. M.; Jayawickrama, D. A.; Webb, A. G.; Sweedler, J. V. Anal. Chem. 2002, 74, $5550-5555$.

(15) Subramanian, R.; Kelley, W. P.; Floyd, P. D.; Tan, Z. J.; Webb, A. G.; Sweedler, J. V. Anal. Chem. 1999, 71, 5335-5339.

(16) Olson, D. L.; Lacey, M. E.; Webb, A. G.; Sweedler, J. V. Anal. Chem. 1999, 71, 3070-3076. 
(17) Ciobanu, L.; Seeber, D. A.; Pennington, C. H. J. Mag. Res. 2002, 158, 178-182.

(18) Hertzog, D. E.; Michalet, X.; Jager, M.; Kong, X.; Santiago, J. G.; Weiss, S.; Bakajin, O. Anal. Chem. 2004, 76, 7169-7178.

(19) Song, H.; Ismagilov, R. F. J. Am. Chem. Soc. 2003, 125, 14613-14619.

(20) Kakuta, M.; Jayawickrama, D. A.; Wolters, A. M.; Manz, A.; Sweedler, J. V. Anal. Chem. 2003, 75, 956-960.

(21) Li, Y.; Wolters, A. M.; Malawey, P. V.; Sweedler, J. V.; Webb, A. G. Anal. Chem. 1999, $71,4815-4820$.

(22) Ciobanu, L.; Jayawickrama, D. A.; Zhang, X.; Webb, A. G.; Sweedler, J. V. Angew Chem. Int. Ed. Engl. 2003, 42, 4669-4672.

(23) Massin, C.; Vincent, F.; Homsy, A.; Ehrmann, K.; Boero, G.; Besse, P.-A.; Daridon, A.; Verpoorte, E.; Rooij, N. F. d.; Popvic, R. S. J. Mag. Res 2003, 164, 242-255.

(24) Wensink, H.; Benito-Lopez, F.; Hermes, D. C.; Verboom, W.; Gardeniers, H. J. G. E.; Reinhoudt, D. N.; Berg, A. v. d. Lab Chip 2005, 5, 280-284.

(25) Walton, J. H.; Ropp, J. S. d.; Shutov, M. V.; Goloshevsky, A. G.; McCarthy, M. J.; Smith, R. L.; Collins, S. D. Anal. Chem. 2003, 75, 5030-5036.

(26) Moule, A. J.; Spence, M. M.; Han, S.-I.; Seeley, J. A.; Pierce, K. L.; Saxena, S.; Pines, A. Proc. Natl. Acad. Sci. USA 2003, 100, 9122-9127.

(27) Seeley, J. A.; Han, S.-I.; Pines, A. J. Magn. Reson. 2004, 167, 282-290.

(28) Olson, D. L.; L. Peck, T.; Webb, A. G.; Magin, R. L.; Sweedler, J. V. Science 1995, 270, 1967-1970.

(29) Olson, D. L.; Lacey, M. E.; Sweedler, J. V. Anal. Chem. 1998, 70, 645-650.

(30) Walker, T. G.; Happer, W. Rev. Mod. Phys. 1997, 69, 629-642. 
(31) Hoult, D. I. Prog. Nucl. Mag. Res. Sp. 1978, 12, 41-77.

(32) Hilty, C.; McDonnell, E.; Granwehr, J.; PIerce, K.; Han, S.-I.; Pines, A. In Preparation.

(33) Woelk, K. J. Mag. Res. 2000, 146, 147-164. 